\title{
Extra variants: 2010-based national population projections
}

\author{
Coverage: UK \\ Date: 23 November 2011 \\ Geographical Area: Country \\ Theme: Population
}

\section{Introduction}

The 2010-based national population projections are published in two parts. The first part was published on 26 October 2011 and included the principal projection and nine standard variant projections. This second release includes 11 further standard 'combination' and special case scenario variants. For a list of all variants available and their publication dates, please see Appendix A.

\section{Background on variant projections}

The principal population projections provide a consistent starting point for all government planning which is affected by the numbers in the population. They are based on assumptions considered to be the best that could be made at the time they are adopted. However, due to the inherent uncertainty of demographic behaviour, any set of projections will inevitably be proved wrong, to a greater or lesser extent, as a forecast of future demographic events or population structure.

To give users of the projections an indication of this uncertainty, a number of variant population projections have also been produced, based on alternative assumptions of future fertility, mortality and migration. In particular, high and low variant assumptions have been produced which are intended as plausible alternatives to the principal assumptions and not to represent upper or lower limits for future demographic behaviour.

It is also sometimes useful to prepare special case scenarios or 'what if' projections, to illustrate the consequences of a particular, but not necessarily realistic, set of assumptions.

\section{Variants available from the 2010-based projections}

The principal projection and nine standard variant projections were published on 26 October 2011. These included six possible 'single component' variants (that is varying only one component at a time from the principal assumptions); two 'combination' variants that produce the largest/smallest 
total population size, and one special case scenario based on an assumption of zero net migration (natural change only).

The following 11 additional standard 'combination' variant projections and special case scenarios are included in this release:

\section{Standard 'combination' variants}

Old age structure

Young age structure

High medium-term dependency ratio

Low medium-term dependency ratio

\section{Special case scenarios}

Replacement fertility

Constant fertility

No mortality improvement

No change

Stationary

No mortality improvement \& zero net migration

Long-term balanced net migration (UK only)

Please see Appendix A for a list of all variants available and their publication dates.

\section{Description of special case scenarios}

\section{Replacement fertility projection}

Replacement fertility is the level of fertility required for the population to replace itself in size in the long-term given constant mortality rates and in the absence of migration. Replacement level is now around 2.075 in the UK, that is, women would need to have, on average, 2.075 children each to ensure the long-term 'natural' replacement of the population. The replacement fertility projection combines assumed replacement level fertility with the principal assumptions of mortality and migration.

Constant fertility projection 
This projection assumes that age specific fertility rates will remain constant at the values assumed for the first year (2010-11) of the principal projection. Although actual age-specific fertility rates for 2010-11 were not known when the principal projection was carried out, the assumed rates were consistent with provisional estimates of total births for the year. Fertility rates have risen since 2002 and a continuation of these 2010-11 fertility rates produces a long-term total fertility rate for the UK of 1.98, which is above that assumed for the principal projection but below that assumed for the standard high fertility variant. The constant fertility projection combines assumed constant level fertility with the principal assumptions of mortality and migration.

No mortality improvement projection

This projection assumes that age/sex specific mortality rates will remain constant at the values assumed for the first year (2010-11) of the principal projection. Although actual age/sex specific mortality rates for 2010-11 were not known when the principal projection was carried out, the assumed rates were consistent with provisional estimates of total deaths for the year. This projection combines assumed no mortality improvement with the principal assumptions of fertility and migration. Table 1-1 below shows period expectation of life in 2034-35 under this scenario compared to principal, high and low standard variants.

\section{Table 1-1}

Period expectation of life at birth in 2034-35, for the standard variants and one special case scenario

Standard variants

High Principal
Special case scenario

No Improvement

Males

England

Wales

Scotland

Northern Ireland

United Kingdom

85.6

83.3

83.6

81.4

78.9

82.8

80.5

77.8

83.3

80.9

78.4

76.1

84.7

82.4

80.1

77.4

\section{Females}

\section{England}

88.7

87.2

85.7

82.8

Wales

88.1

86.6

85.1

82.1

Scotland

86.7

85.1

83.5

86.6

85.1
78.5

\section{Northern Ireland}

88.1

85.1

81.9 


\begin{tabular}{lllll} 
United Kingdom & 88.4 & 87.0 & 85.5 & 82.6 \\
\hline
\end{tabular}

Table source: Office for National Statistics

Download table

XLS XLS format

$(26.5 \mathrm{~Kb})$

Zero net migration (natural change only) projection (published 26 October 2011)

This projection uses the principal assumptions of fertility and mortality and assumes that there will be zero net migration (at every age). It therefore shows the consequences of the principal assumptions of fertility and mortality in the absence of migration, or where migration inflows and outflows are exactly equal at every age.

\section{No change projection}

This projection shows what would happen if fertility, mortality and net migration were to remain constant at current levels. It therefore assumes the fertility rates from the constant fertility projection and the mortality rates from the no mortality improvement projection. Given the fluctuating nature of net migration, it is much more difficult to define what is meant by the current level of net migration. However the long-term assumption in the principal projections is based on analysis of recent trends and, for the UK as a whole, is similar to recent levels of net migration. So the principal migration assumptions have been used for the no change projection.

\section{Stationary projection}

A stationary population has an unchanging size and age structure and arises, eventually, given replacement level fertility, constant mortality rates at all ages and zero net migration at all ages. This projection therefore assumes the fertility rates from the replacement fertility projection, the mortality rates from the no mortality improvement projection and zero net migration at each age as in the zero net migration projection. The extent to which the population is not stationary under these conditions in the short and medium-term reflects inherent 'population momentum' resulting from the existing population age structure.

No mortality improvement \& zero net migration projection

This projection uses the fertility rates from the principal projection, the mortality rates from the no mortality improvement projection and zero net migration at each age as in the zero net migration projection. It enables the effects of the principal fertility and mortality assumptions on future population growth to be distinguished from each other:

- The no mortality improvement \& zero net migration projection differs from the stationary projection only in the fertility assumptions used. A comparison of these variants therefore shows the impact of below replacement level fertility (as assumed in the principal projection) on future population change.

- Similarly, the no mortality improvement \& zero net migration projection differs from the zero net migration projection only in the mortality assumptions used. A comparison of these variants 
therefore shows the impact of mortality improvement (as assumed in the principal projection) on future population change.

\section{Long-term balanced net migration projection}

This projection assumes that total net migration will decline to zero in the long-term, with inmigration and out-migration total flows being equal from 2037 onwards. However, unlike the zero net migration variant, it is not assumed that inflows and outflows will be equal at every age in the longterm. This variant is only produced for the UK.

\section{Results}

Please note that the data presented in this report does not reflect further changes to the state pension age published by the government in the Pensions Bill 2011, which received Royal Assent on 3 November 2011. The Act puts into law changes to the state pension age timetable. From April 2016, women's state pension age will rise faster than originally planned, equalising with men's at 65 by November 2018. Between December 2018 and October 2020, men and women's state pension ages will be increased from 65 to 66 .

Further information relating to these changes can be found on the Department for Work and Pensions (DWP) website at: http://www.dwp.gov.uk/policy/pensions-reform/ under pensions reform, which includes the new state pension age timetable at: http://www.dwp.gov.uk/docs/spatimetable.pdf

Table 1-2 presents projections of the total population under the principal projection, standard variants and special case scenarios for the UK in 2035, 2060, 2085 and 2110. Data for the constituent countries and three additional measures, the percentage of the population under 16 , percentage of population 65 and over and dependants per 1000 persons of working age (dependency ratios), are available in the data download.

\section{Table 1-2}


Measures of population structure under the principal projection, standard variant projections and special case scenarios, UK 2035, 2060, 2085 and 2110

Total population (000s)

$(2010=62,262)$

2035

2060

2085

2110

Principal projection

73,208

81,481

89,300

96,979

SINGLE COMPONENT VARIANTS

High fertility

75,048

87,050

100,533

115,579

Low fertility

70,832

75,380

78,277

80,395

High life expectancy

73,920

84,637

95,522

106,843

Low life expectancy

72,467

78,181

82,777

86,686

High migration

75,135

85,811

96,120

106,178

Low migration

71,280

77,152

82,480

87,780

COMBINATION VARIANTS

High/low population variants

High fertility, High life

77,746

94,817

114,471

136,791

expectancy, High migration

68,215

68,021

65,879

63,415

expectancy, Low migration

Youngest/ oldest age structure

High fertility, Low life

76,283

88,202

100,864

113,934

expectancy, High migration

Low fertility, High life

69,658

74,314

77,732

80,682

expectancy, Low migration

Highest/ lowest medium-term dependency ratios

High fertility, High life

73,778

85,620

99,188

114,935

expectancy, Low migration 
Low fertility, Low life

expectancy, High migration

SPECIAL CASE SCENARIOS

Replacement fertility

75,124

87,525

101,815

118,099

Constant fertility

No mortality improvement

Zero net migration (natural

change only)

No change

Constant fertility, No mortality

improvement

\section{Stationary projection}

Replacement fertility, No

mortality improvement, Zero net

migration

No mortality improvement \&

Zero net migration

74,048

84,458

95,745

75,776

80,079

64,073

60,813

65,740

71,695

78,737

86,433

94,271

65,045

63,526

62,900

62,988 83,676

57,861 
Actual and projected percentage of the population aged 65 and over, United Kingdom, 1981-2085

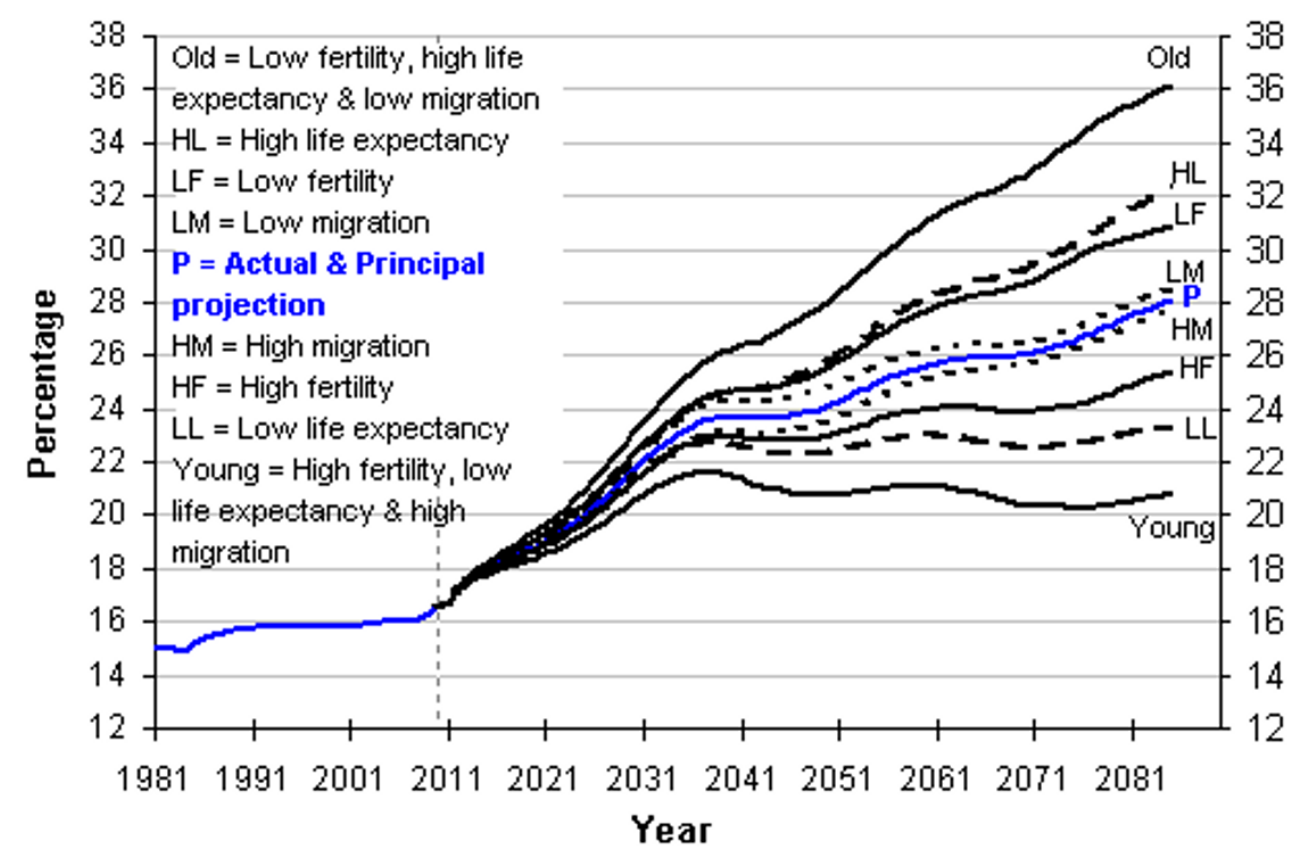

\section{Download chart}

GIF GIF format

$(12 \mathrm{~Kb})$

\section{Download chart}

XLS XLS format

$(39 \mathrm{~Kb})$

The equivalent charts for the constituent countries of the UK can be found in Appendix B. 


\section{Appendices}

Appendix A: Principal and variant projections with associated assumptions and availability Published Projections

\begin{tabular}{lrrrrr}
\hline & & Fertility & Life expectancy & $\begin{array}{r}\text { Net } \\
\text { migration }\end{array}$ & Availability \\
1 & $\begin{array}{l}\text { Principal projection } \\
\text { Standard 'single component' variants }\end{array}$ & Principal & Principal & Principal & 26 Oct 2011 \\
2 & High fertility & High & Principal & Principal & 26 Oct 2011 \\
3 & Low fertility & Low & Principal & Principal & 26 Oct 2011 \\
4 & High life expectancy & Principal & High & Principal & 26 Oct 2011 \\
5 & Low life expectancy & Principal & Low & Principal & 26 Oct 2011 \\
6 & High migration & Principal & Principal & High & 26 Oct 2011 \\
7 & Low migration & Principal & Principal & Low & 26 Oct 2011
\end{tabular}

\section{Standard 'combination' variants}

$\begin{array}{llr}8 & \text { High population } & \text { High } \\ 9 & \text { Low population } & \text { Low } \\ 10 & \text { Young age structure } & \text { High } \\ 11 & \text { Old age structure } & \text { Low } \\ 12 & \begin{array}{l}\text { High medium-term } \\ \text { dependency } \\ 13\end{array} & \text { High } \\ & \begin{array}{l}\text { Low medium-term } \\ \text { dependency }\end{array} & \text { Low }\end{array}$

\section{Special case scenarios}

14 Replacement fertilifyeplacement

15 Constant fertility Constant

$16 \quad$ No mortality improvement (natural change only)

No change

Stationary
Zero net migration Principal
Constant

Principal

Replacement
High

High

26 Oct 2011

Low

Low

26 Oct 2011

Low High

High Low

High Low

Low High

23 Nov 2011

23 Nov 2011

23 Nov 2011

23 Nov 2011

Principal Principal

23 Nov 2011

Principal Principal

23 Nov 2011

No improvement Principal

23 Nov 2011

Principal Zero

26 Oct 2011

23 Nov 2011

23 Nov 2011 

net migration

Table source: Office for National Statistics

\section{Download table}

XLS XLS format

$(27.5 \mathrm{~Kb})$

Appendix B: Charts - Population aged 65 \& over for the constituent countries

Actual and projected percentage of the population aged 65 and over, England, 1981-2085

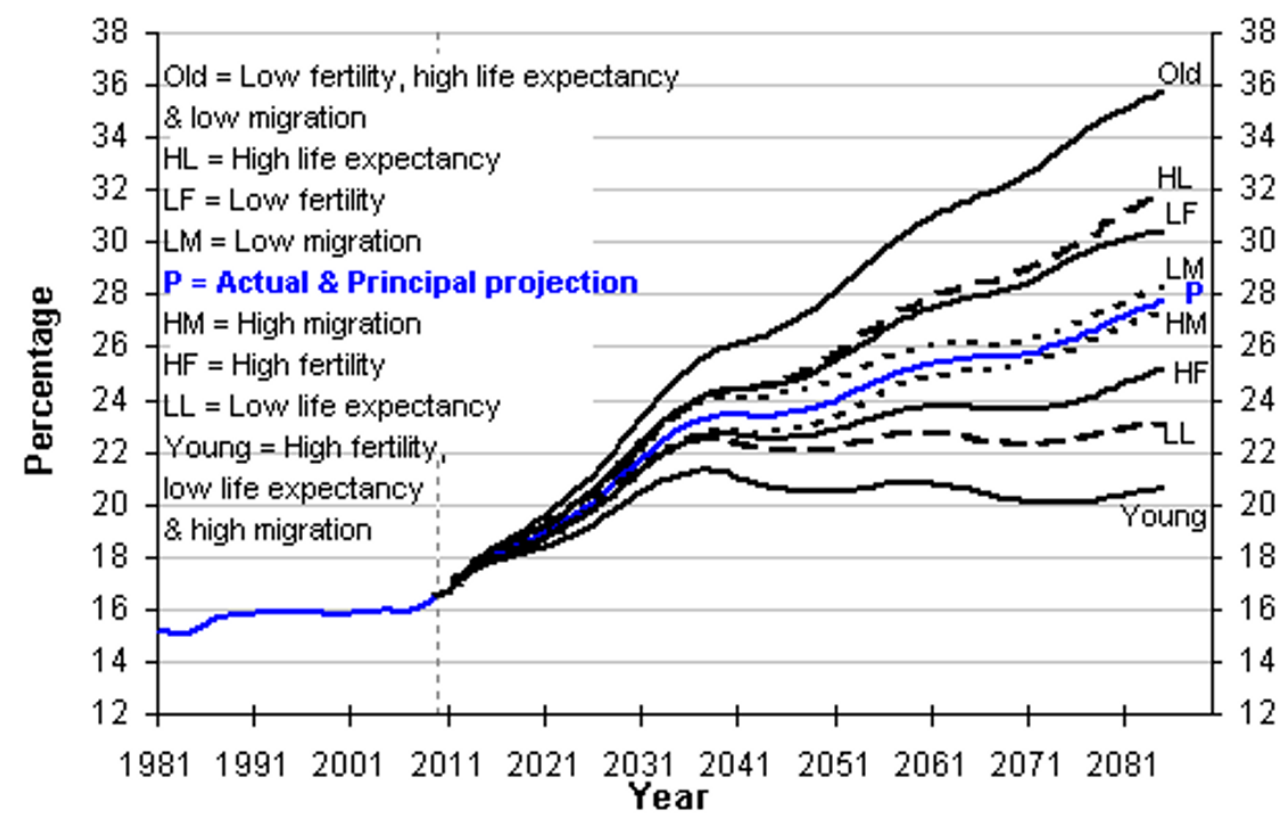

\section{Download chart}

GIF GIF format

$(12 \mathrm{~Kb})$

\section{Download chart}

\section{XLS XLS format}

$(41.5 \mathrm{~Kb})$ 
Actual and projected percentage of the population aged 65 and over, Wales, 1981-2085

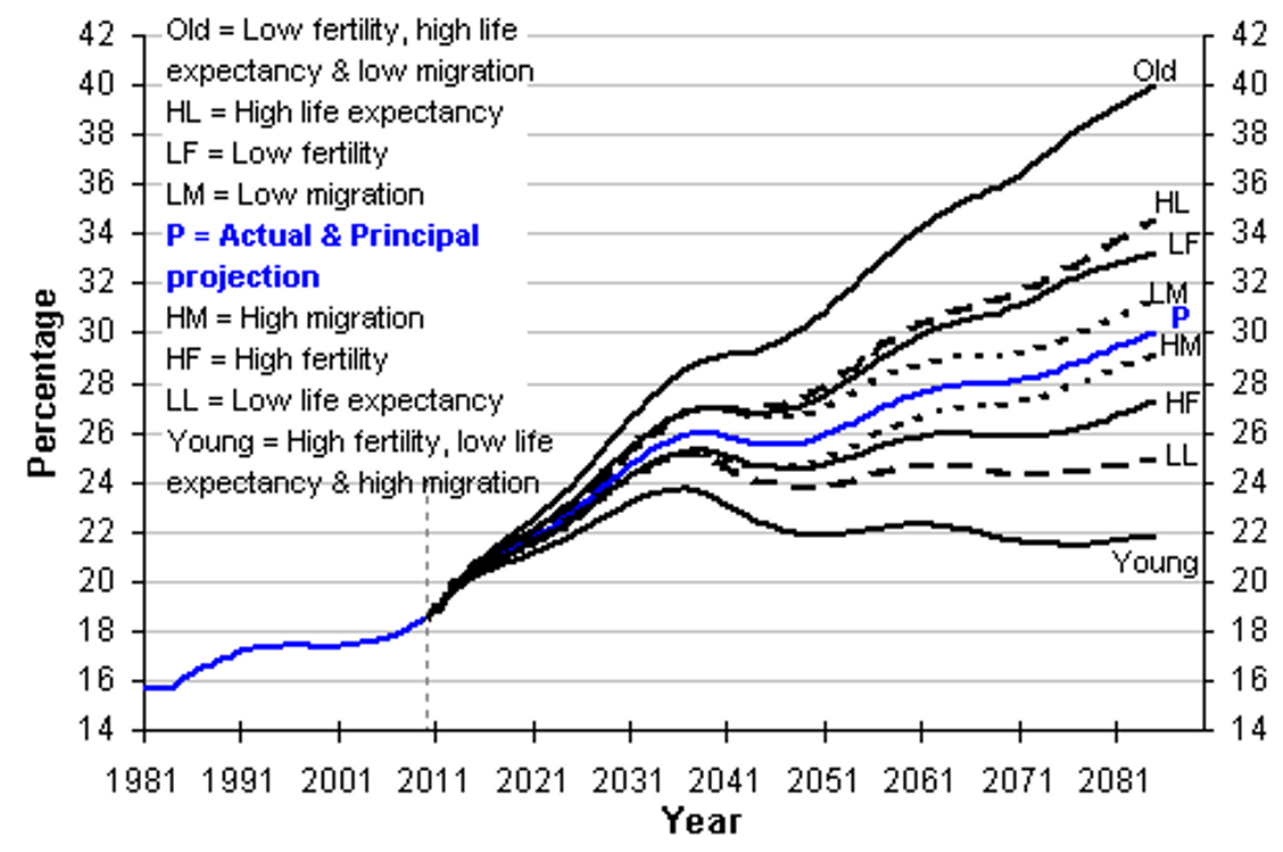

\section{Download chart}

\section{GIF GIF format}

(11.9 Kb)

\section{Download chart}

\section{XLS XLS format} (39.5 Kb) 
Actual and projected percentage of the population aged 65 and over, Scotland, 1981-2085

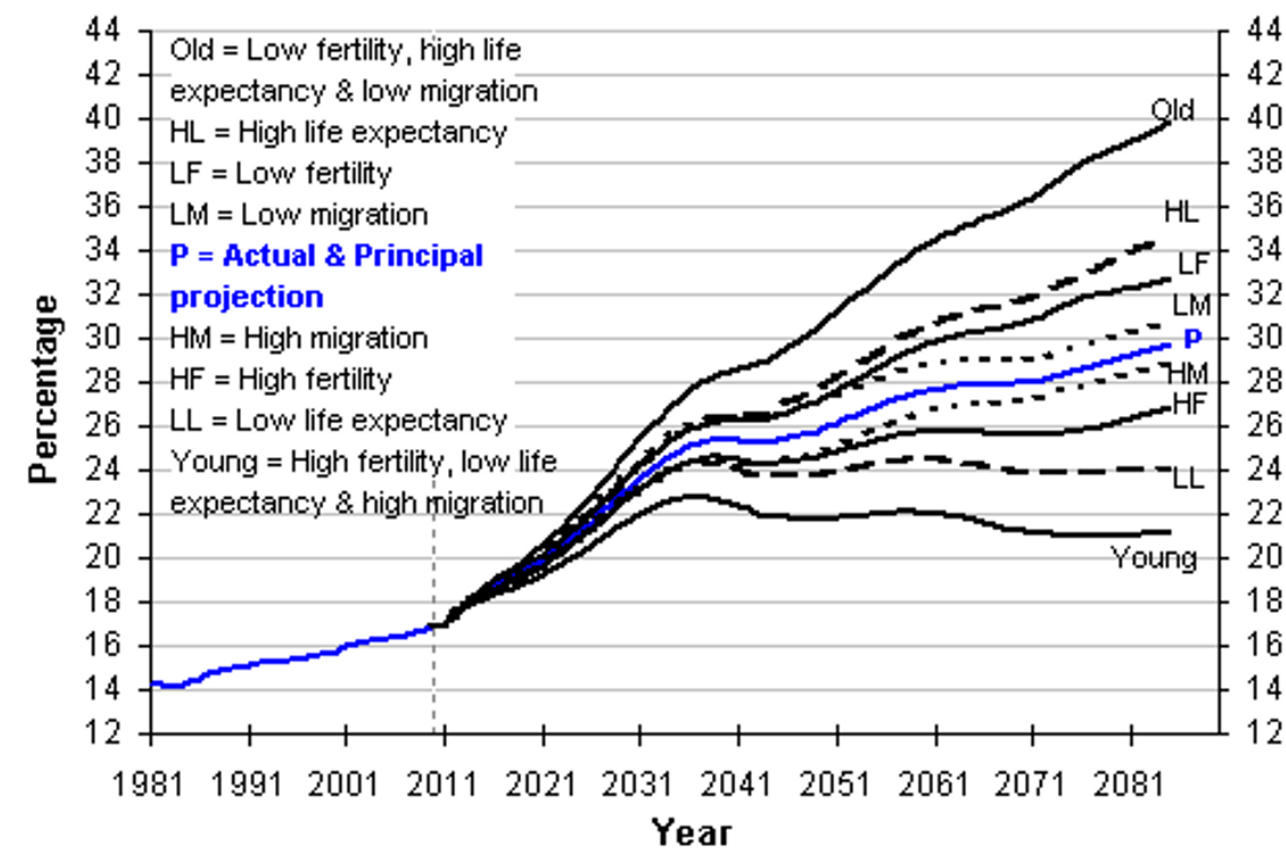

\section{Download chart}

\section{GIF GIF format}

$(15.7 \mathrm{~Kb})$

\section{Download chart}

\section{XLS XLS format}

(39 Kb) 
Actual and projected percentage of the population aged 65 and over, Northern Ireland, 1981-2085

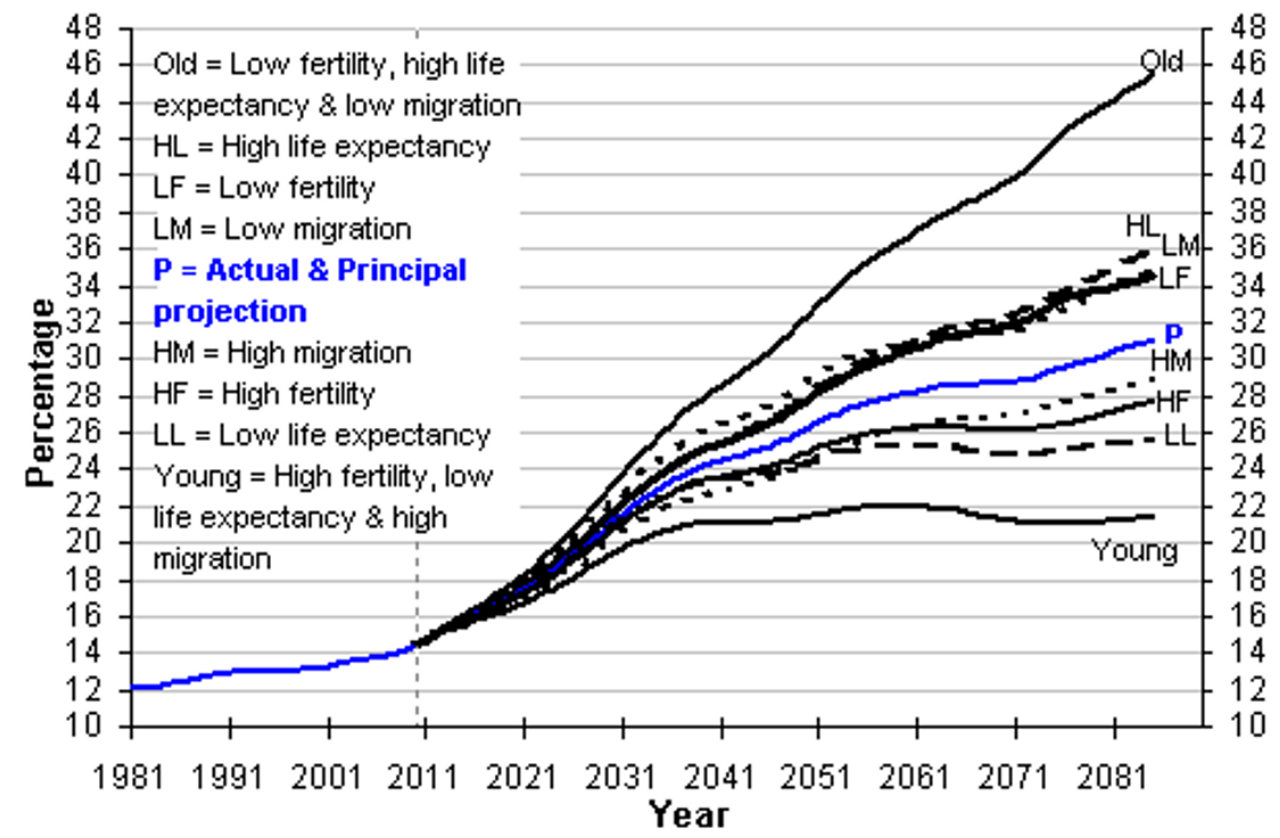

\section{Download chart}

GIF GIF format

$(12.7 \mathrm{~Kb})$

\section{Download chart}

XLS XLS format

$(39 \mathrm{~Kb})$

\section{Appendix C: Charts - National population projections expert advisory panel}

An expert academic panel advises the ONS on the appropriate assumptions to use for the national population projections. A list of membership and a note of the panel meeting held on the 17 March 2011 can be found in the October 2011 release in the background and methodology report. The charts in this appendix give details of the panel's views on the most likely levels of the total fertility rate (TFR), life expectancy at birth and total net migration to the UK (and associated 67 per cent and 95 per cent confidence intervals) for the years 2014 and 2034. 
Respondent's estimates of the TFR in 2014 (and associated 67\% confidence intervals)

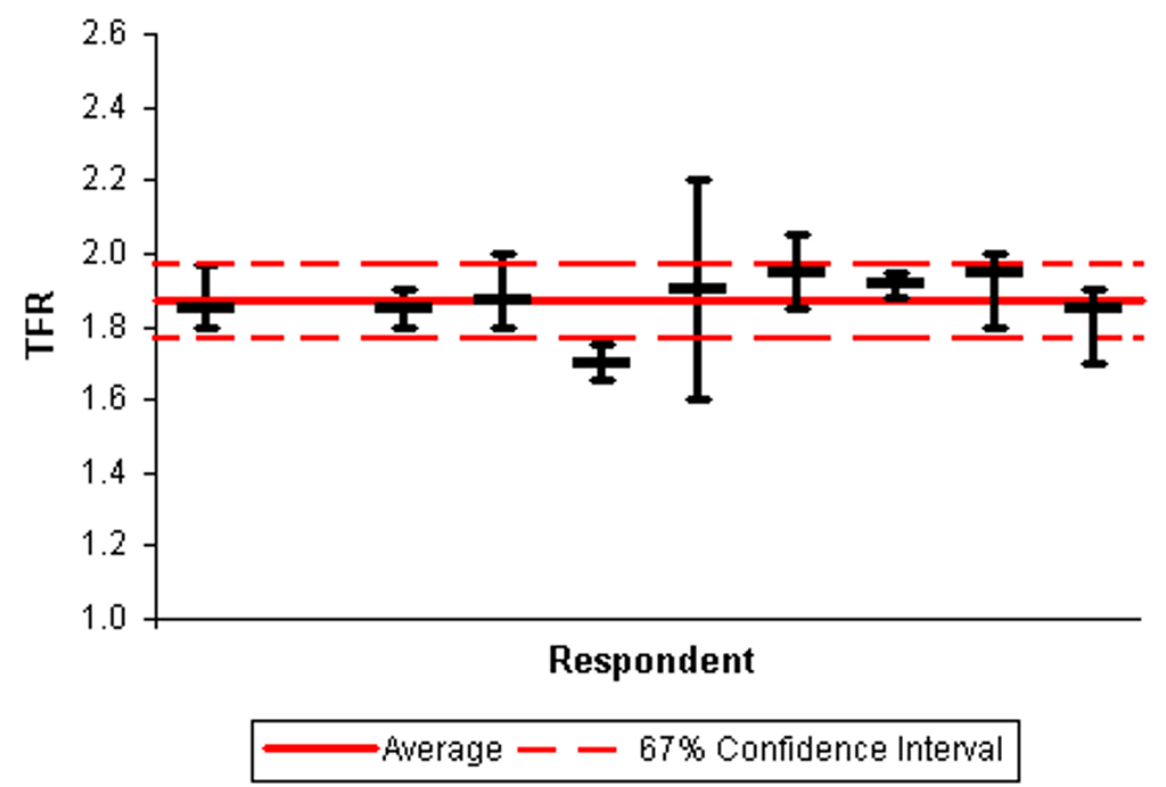

\section{Download chart}

\section{GIF GIF format}

(4.9 Kb)

Download chart

\section{XLS XLS format}

(18.5 Kb) 
Respondent's estimates of the TFR in 2034 (and associated 67\% confidence intervals)

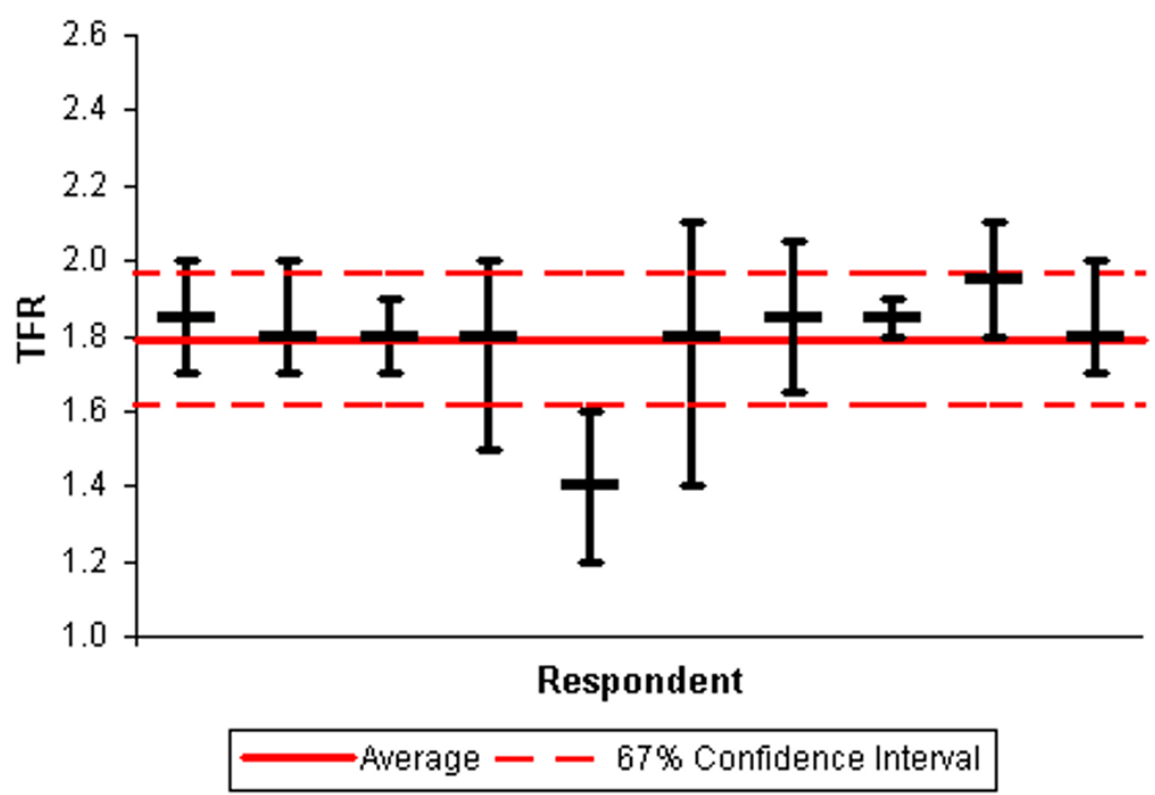

Download chart

GIF GIF format

$(5.5 \mathrm{~Kb})$

Download chart

XLS XLS format

$(18 \mathrm{~Kb})$ 
Respondent's estimates of period life expectancy for males in 2014 (and associated 67\% confidence intervals)

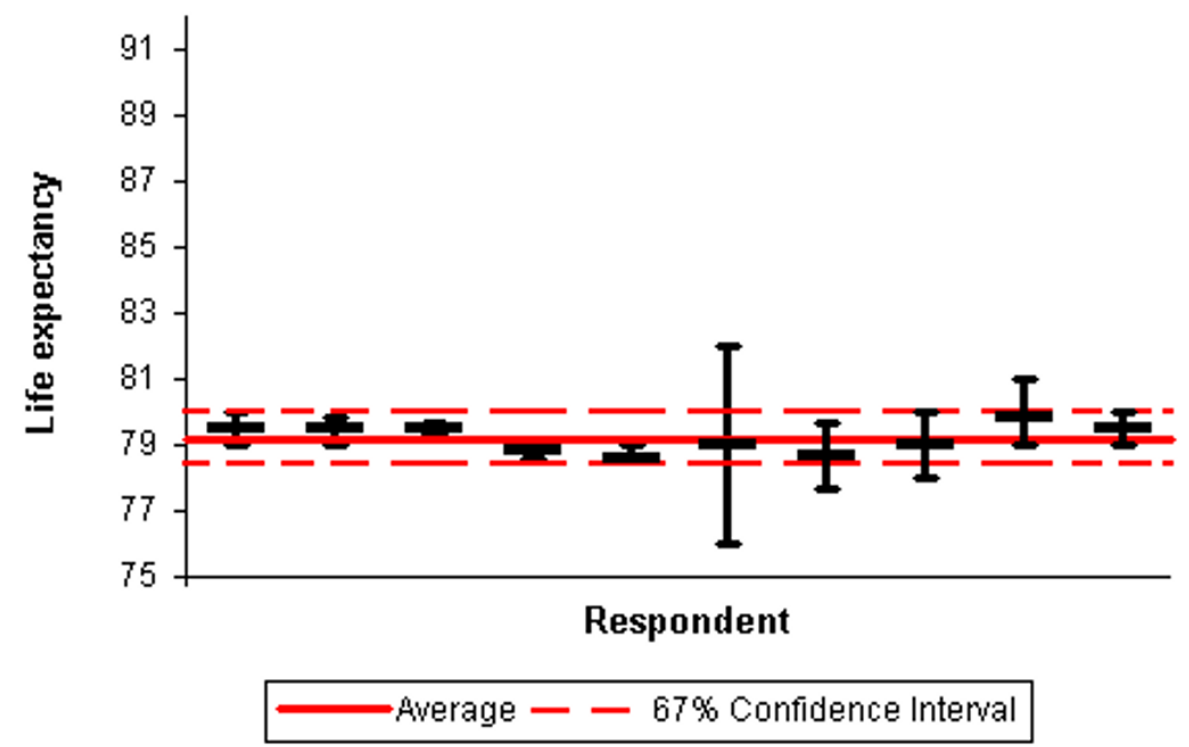

\section{Download chart}

GIF GIF format

$(5.1 \mathrm{~Kb})$

Download chart

XLS XLS format

$(18.5 \mathrm{~Kb})$ 
Respondent's estimates of period life expectancy for males in 2034 (and associated 67\% confidence intervals)

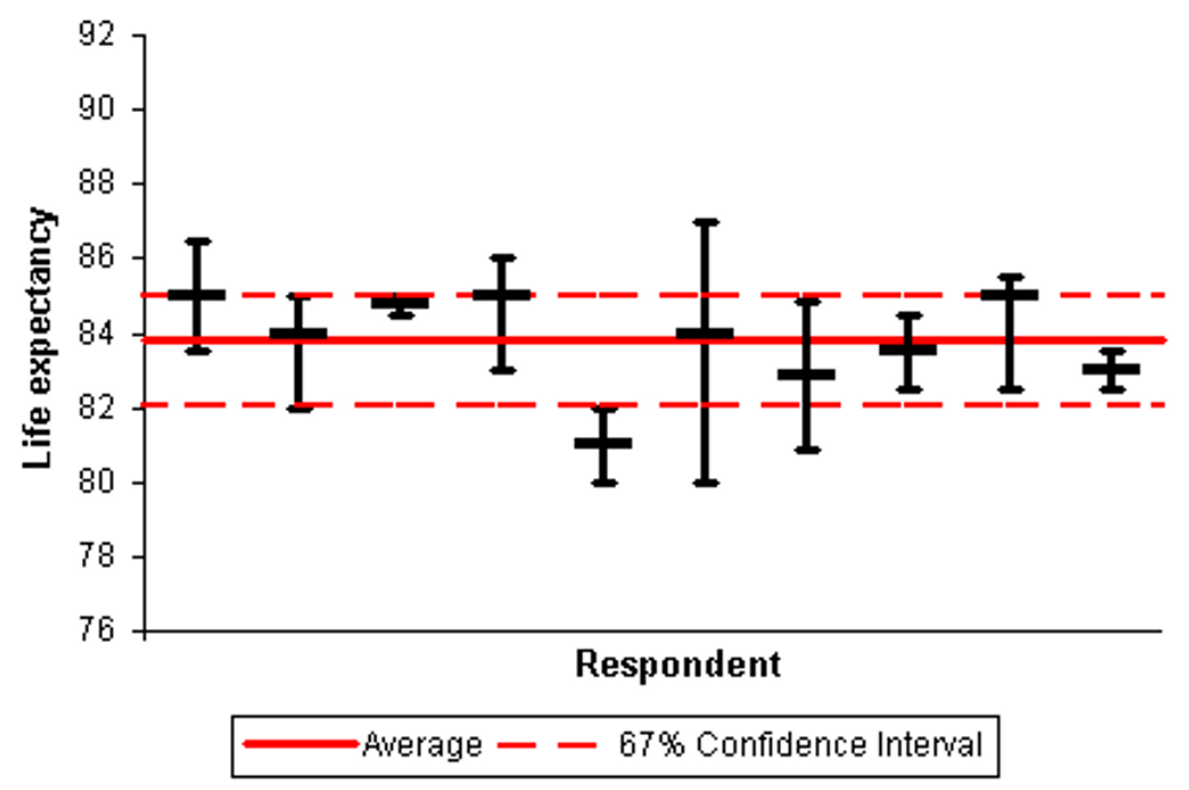

\section{Download chart}

GIF GIF format

$(5.9 \mathrm{~Kb})$

Download chart

XLS XLS format

$(18.5 \mathrm{~Kb})$ 
Respondent's estimates of period life expectancy for females in 2014 (and associated 67\% confidence intervals)

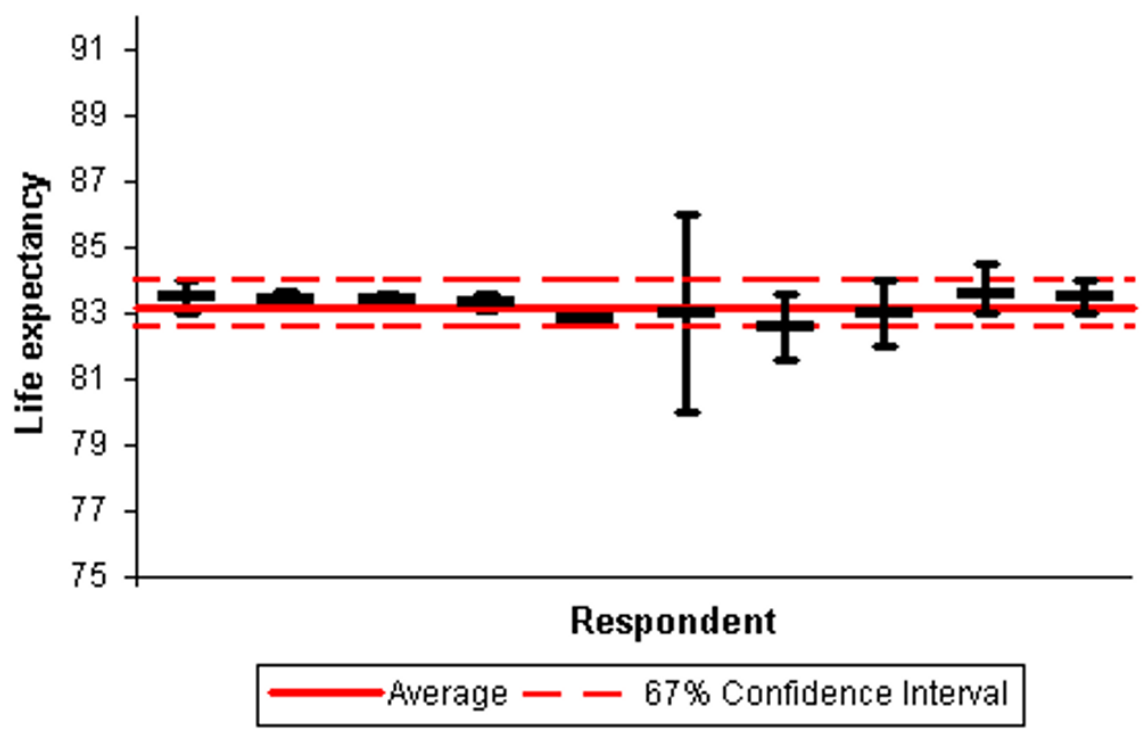

Download chart

GIF GIF format

(5.2 Kb)

Download chart

XLS XLS format

(18.5 Kb) 
Respondent's estimates of period life expectancy for females in 2034 (and associated 67\% confidence intervals)

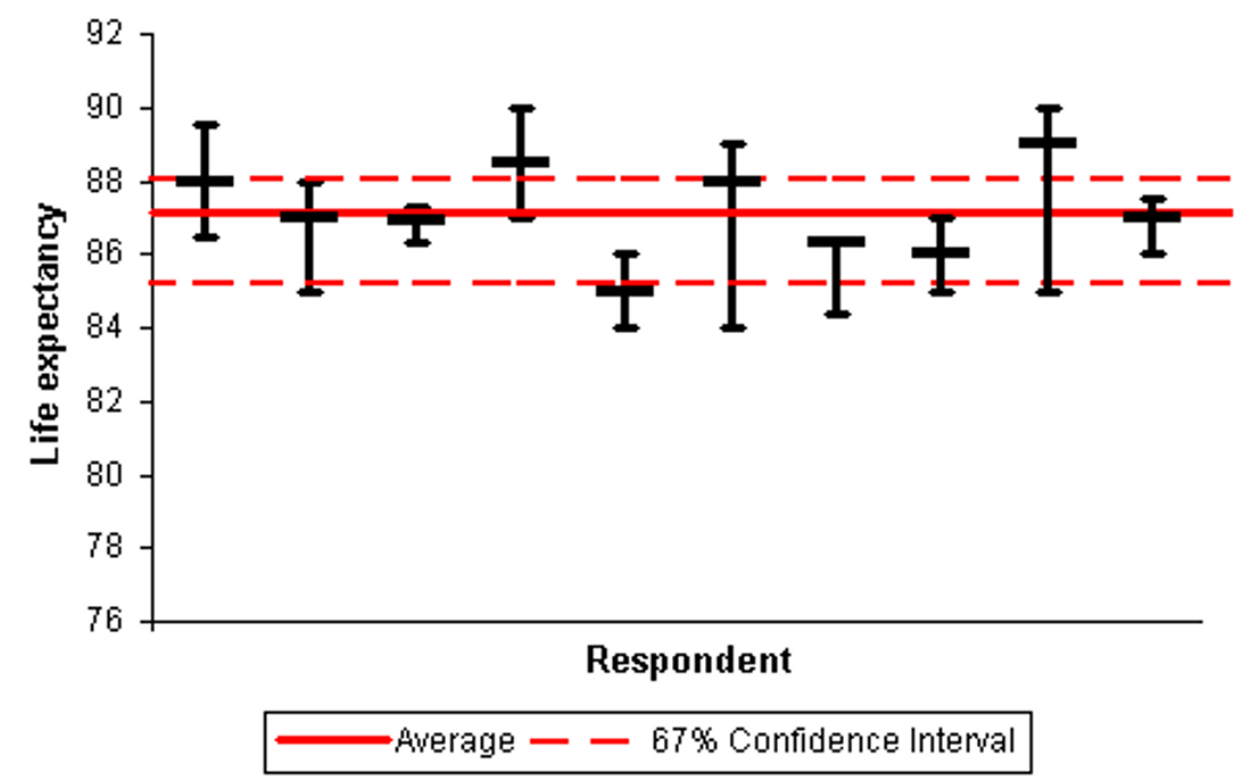

\section{Download chart}

GIF GIF format

$(5.9 \mathrm{~Kb})$

Download chart

XLS XLS format

$(18.5 \mathrm{~Kb})$ 
Respondent's estimates of total net migration in 2014 (and associated 67\% confidence intervals)

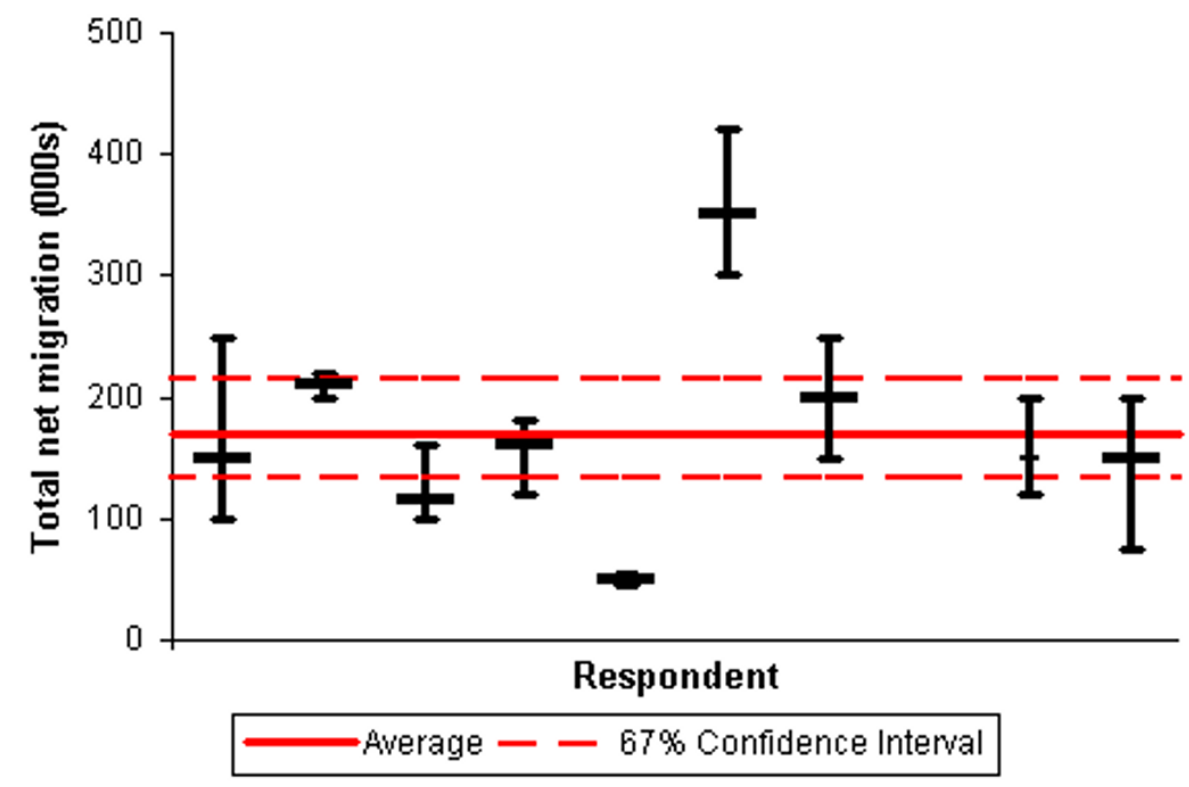

\section{Download chart}

GIF GIF format

(5.4 Kb)

Download chart

XLS XLS format

$(18.5 \mathrm{~Kb})$ 
Respondent's estimates of total net migration in 2034 (and associated 67\% confidence intervals)

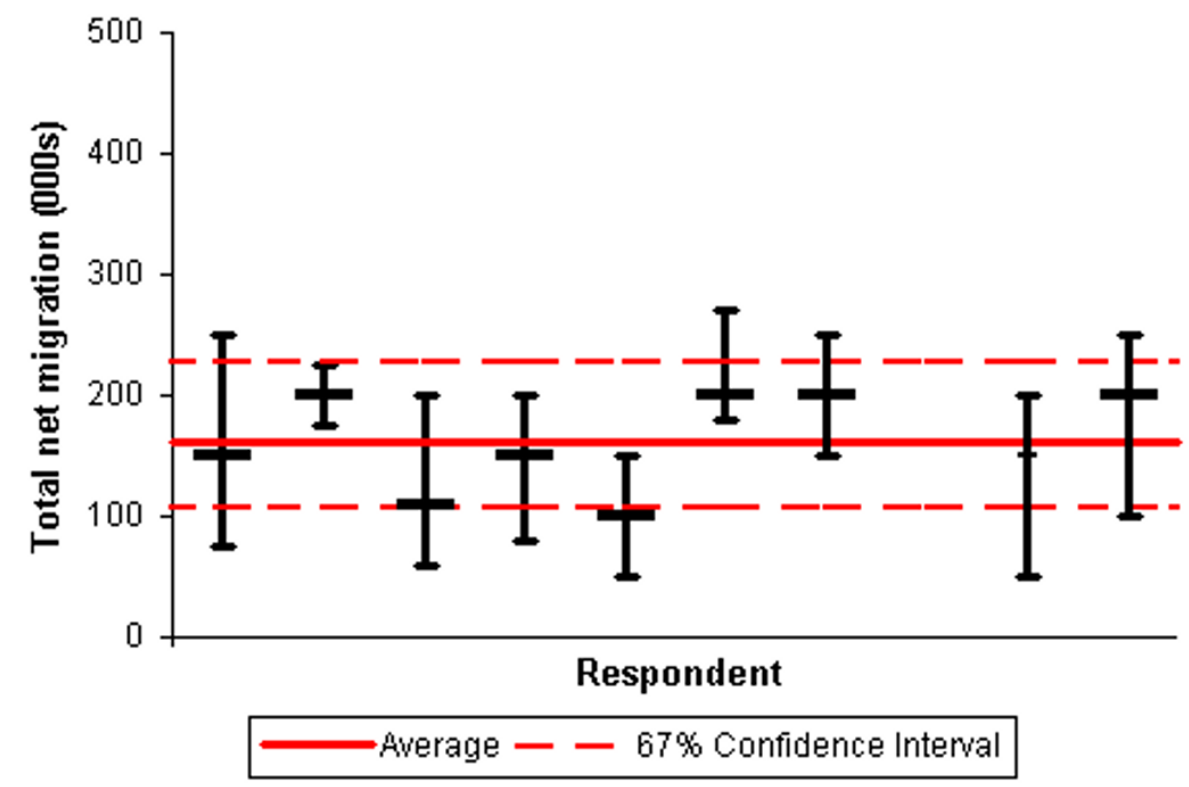

\section{Download chart}

GIF GIF format

(6.1 Kb)

Download chart

XLS XLS format

(19 Kb) 
Respondent's estimates of the TFR in 2014 (and associated 95\% confidence intervals)

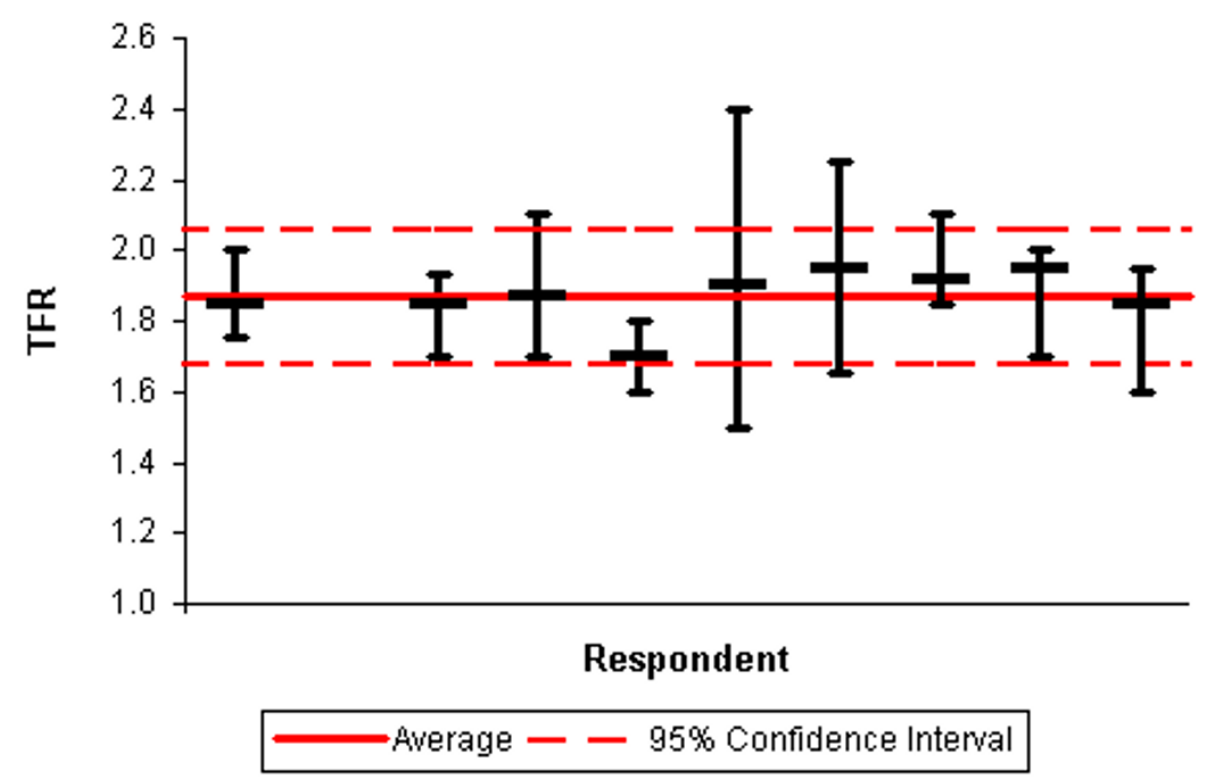

\section{Download chart}

GIF GIF format

(5.4 Kb)

Download chart

XLS XLS format

$(18 \mathrm{~Kb})$ 
Respondent's estimates of the TFR in 2034 (and associated 95\% confidence intervals)

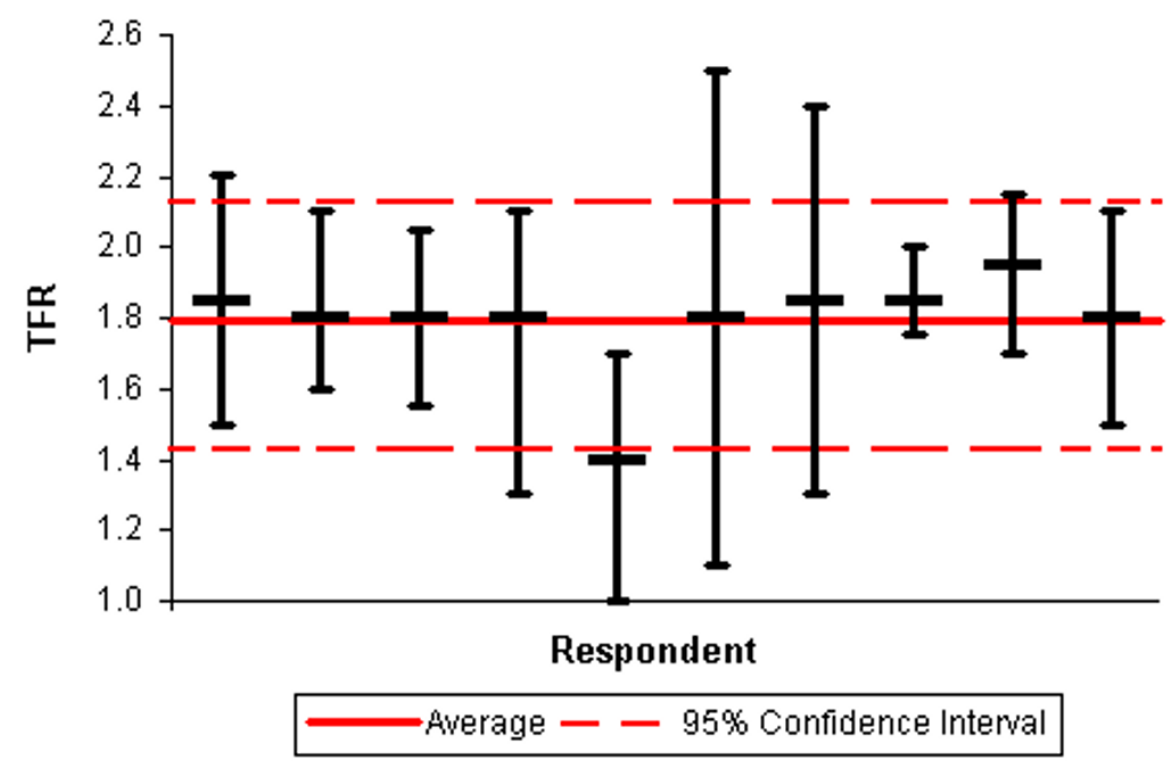

\section{Download chart}

\section{GIF GIF format}

$(6.8 \mathrm{~Kb})$

\section{Download chart}

\section{XLS XLS format}

$(18 \mathrm{~Kb})$ 
Respondent's estimates of period life expectancy for males in 2014 (and associated 95\% confidence intervals)

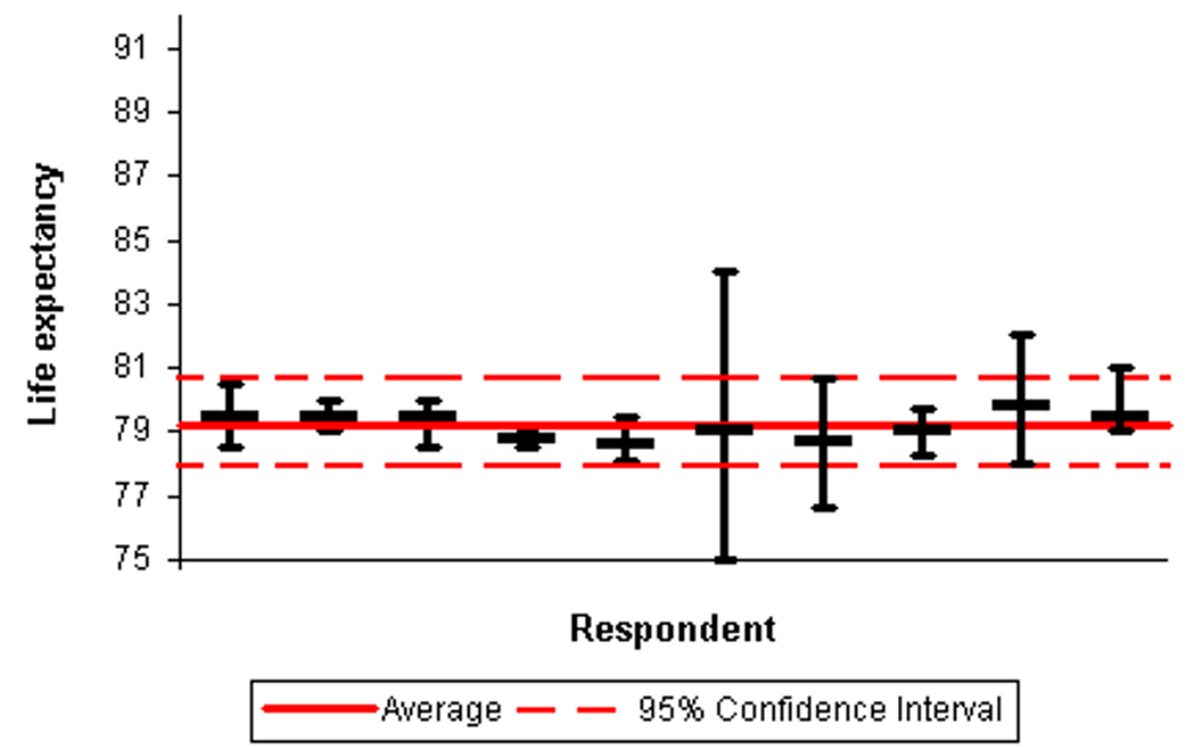

\section{Download chart}

GIF GIF format

(5.7 Kb)

Download chart

XLS XLS format

$(18 \mathrm{~Kb})$ 
Respondent's estimates of period life expectancy for males in 2034 (and associated 95\% confidence intervals)

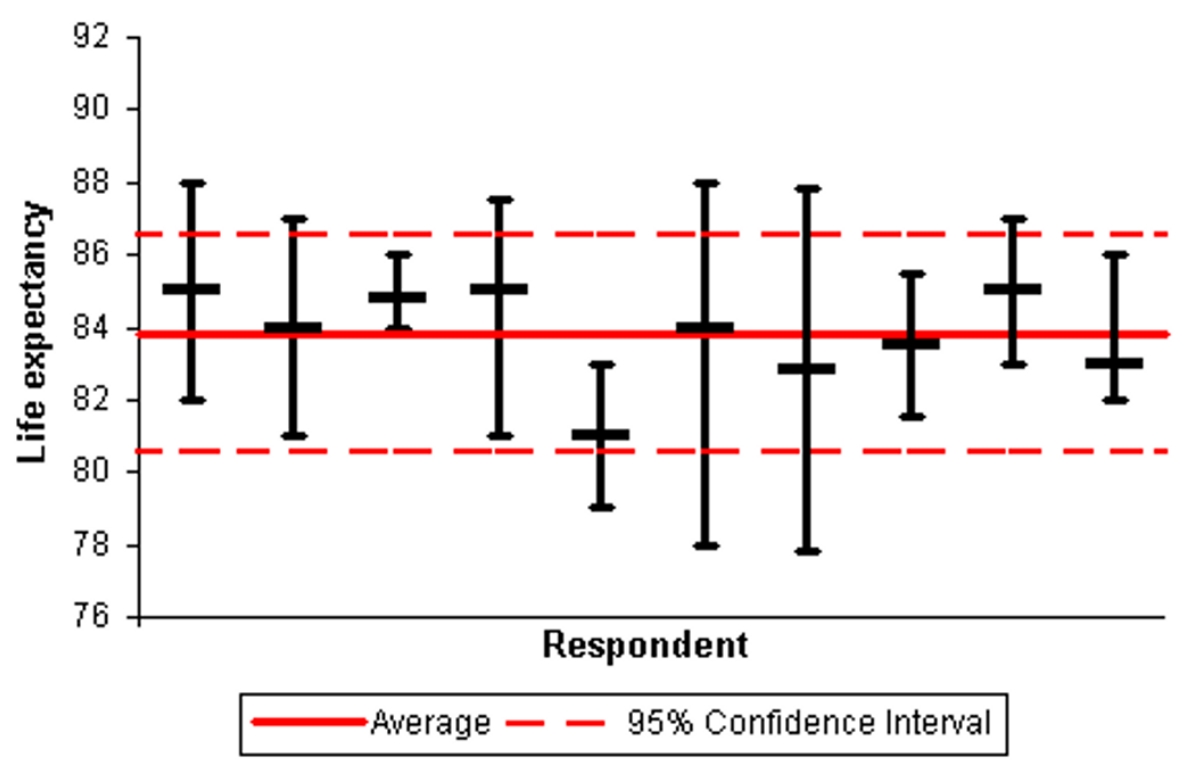

\section{Download chart}

GIF GIF format

$(6.8 \mathrm{~Kb})$

Download chart

XLS XLS format

$(18.5 \mathrm{~Kb})$ 
Respondent's estimates of period life expectancy for females in 2014 (and associated 95\% confidence intervals)

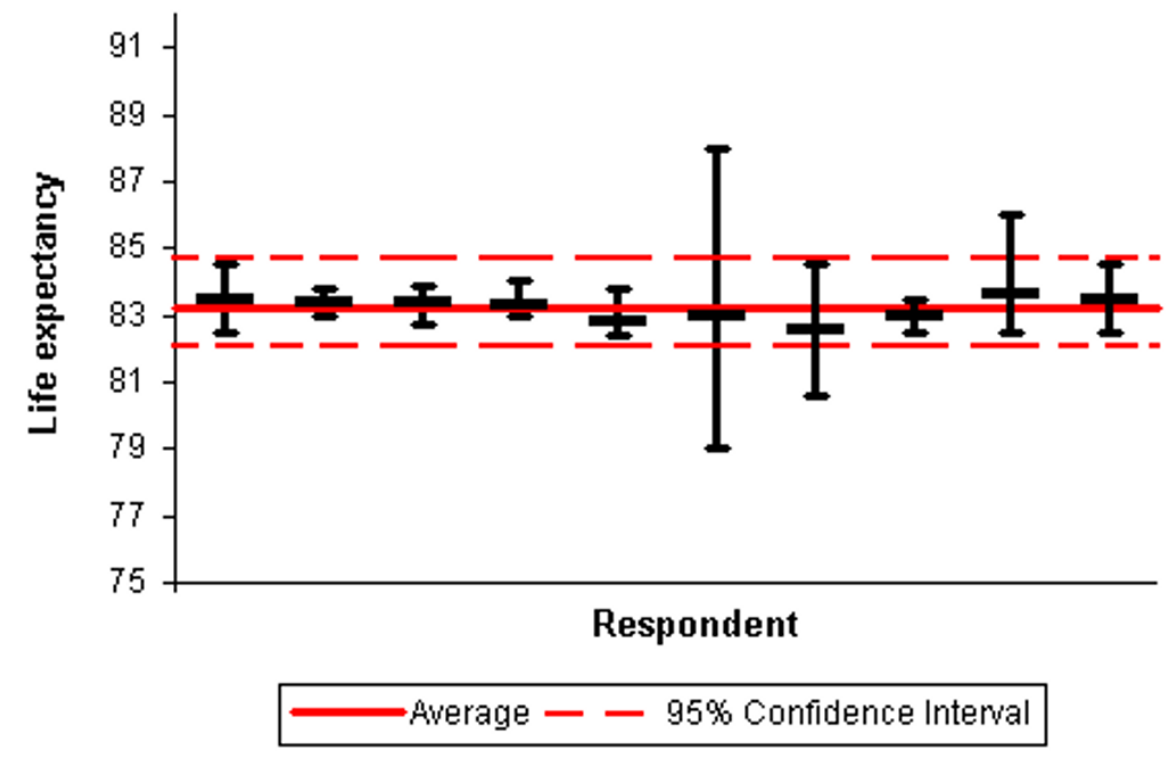

Download chart

\section{GIF GIF format}

$(5.5 \mathrm{~Kb})$

Download chart

\section{XLS XLS format}

$(18 \mathrm{~Kb})$ 
Respondent's estimates of period life expectancy for females in 2034 (and associated 95\% confidence intervals)

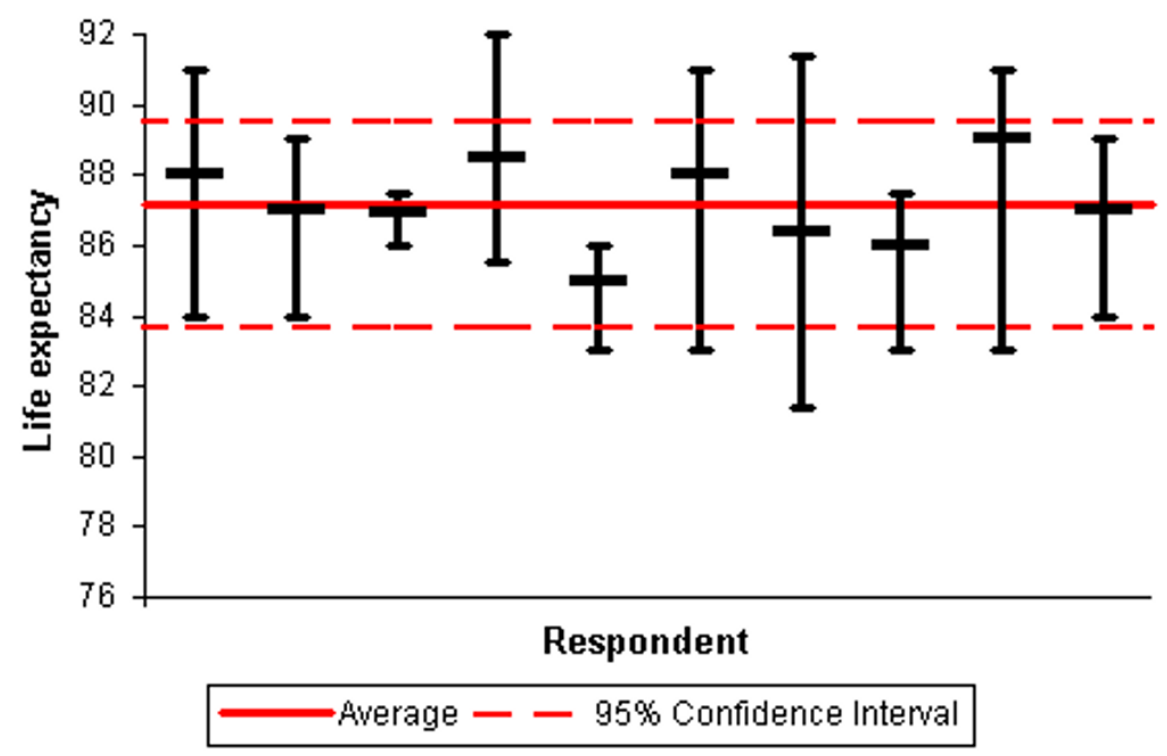

\section{Download chart}

\section{GIF GIF format}

$(7 \mathrm{~Kb})$

\section{Download chart}

\section{XLS XLS format}

$(18 \mathrm{~Kb})$ 
Respondent's estimates of total net migration in 2014 (and associated 95\% confidence intervals)

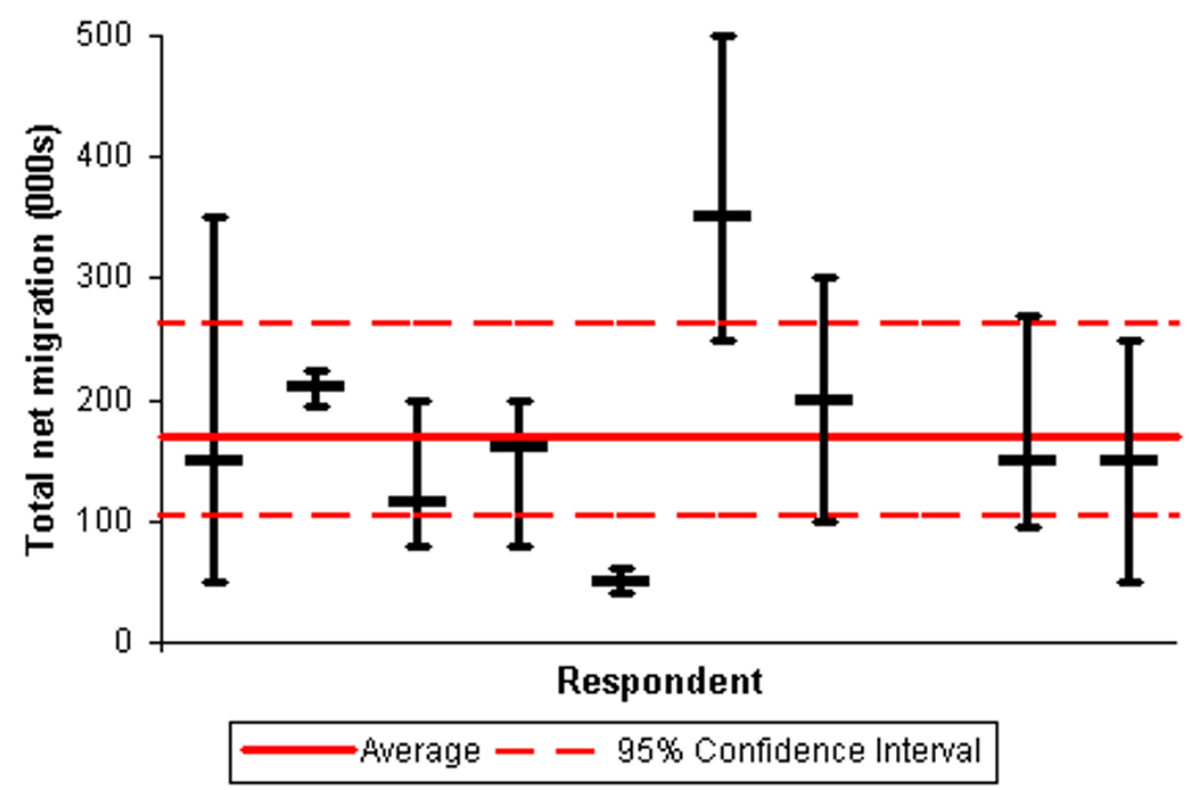

\section{Download chart}

GIF GIF format

$(6.3 \mathrm{~Kb})$

Download chart

XLS XLS format

$(18 \mathrm{~Kb})$ 
Respondent's estimates of total net migration in 2034 (and associated 95\% confidence intervals)

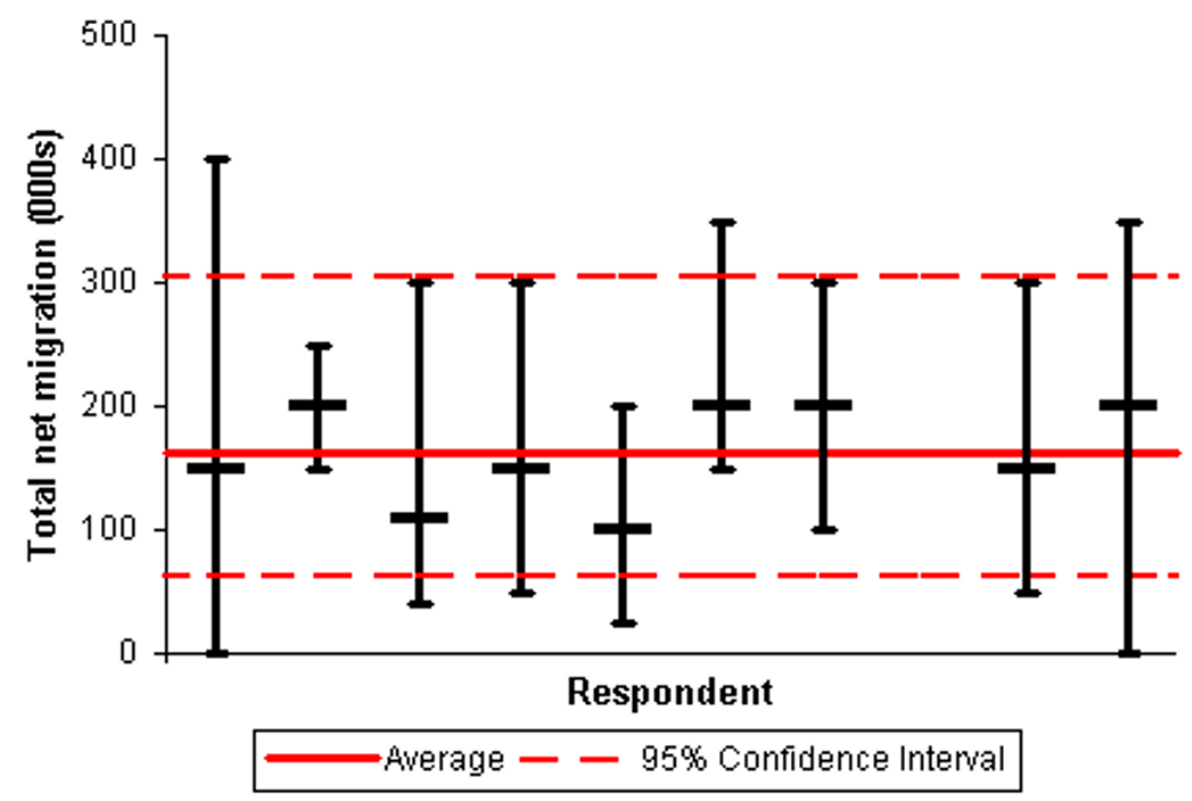

\section{Download chart}

\section{GIF GIF format}

$(7.5 \mathrm{~Kb})$

Download chart

\section{XLS XLS format}

$(18 \mathrm{~Kb})$ 